\section{BMJ Open}

Ophthalmology

\title{
Retinal arteriolar oxygen saturation predicts the need for intravitreal aflibercept in patients with diabetic macular oedema
}

\author{
Søren Leer Blindbæk (1) , ${ }^{1,2,3}$ Tunde Peto, ${ }^{2,4}$ Jakob Grauslund ${ }^{1,2,5}$
}

To cite: Blindbæk SL, Peto T, Grauslund J. Retinal arteriolar oxygen saturation predicts the need for intravitreal aflibercept in patients with diabetic macular oedema. BMJ Open Ophthalmology 2020;5:e000382. doi:10.1136/ bmjophth-2019-000382

Received 7 August 2019 Revised 7 0ctober 2019 Accepted 9 December 2019
Check for updates

C) Author(s) (or their employer(s)) 2020. Re-use permitted under CC BY-NC. No commercial re-use. See rights and permissions. Published by BMJ.

${ }^{1}$ Department of Ophthalmology, Odense University Hospital, Odense, Denmark

${ }^{2}$ Department of Clinical Research, University of Southern Denmark, Odense, Denmark ${ }^{3}$ OPEN, Open Patient data Explorative Network, Odense University Hospital, Odense, Denmark

${ }^{4}$ Centre for Public Health, Queen's University Belfast, Belfast, United Kingdom

${ }^{5}$ Steno Diabetes Center Odense, Odense, Denmark

Correspondence to Dr Søren Leer Blindbæk; Soeren. leer.blindbaek@rsyd.dk

\section{ABSTRACT}

Objective Given the increasing burden of repetitive intravitreal injections in diabetic macular oedema (DMO) treatment, non-invasive markers of treatment outcome are needed. Hence, we aimed to examine retinal oximetry parameters as markers of need for intravitreal aflibercept in patients with DMO.

Methods This study was based on data from a 12-month clinical trial including 35 eyes of 25 patients with centre involving DM0. Retinal oximetry, visual acuity (VA) and central retinal thickness (CRT) were performed at baseline (BL). Patients then received 3 monthly injections of aflibercept followed by focal/grid laser photocoagulation. From month 4 (M4) through 12 (M12), patients were followed monthly and additional injections were given pro re nata if criteria of retreatment were met. We evaluated the difference in need for intravitreal aflibercept in groups of eyes with the highest and lowest retinal arteriolar and venular oxygen saturations, respectively.

Results From BL-M12, overall VA letter score improved by 8.7 (7.2-10.2). Likewise CRT reduced by 100.7 (68.2-133.3) $\mu \mathrm{m}$ and the mean number of injections was 4.3 (3.8-4.8). Overall retinal arteriolar and venular oxygen saturations were 95.7 (93.0-98.4)\% and 62.7 $(59.4-65.9) \%$ at BL. Eyes with the highest retinal arteriolar oxygen saturations had significantly more injections between BL and M12 compared with eyes with the lowest retinal arteriolar oxygen saturations $(5.0(4.2-5.8)$ vs 3.6 (3.1-4.0), $p=0.002$ ).

Conclusion Higher retinal arteriolar oxygen saturation independently predicted the need for more intravitreal aflibercept during the first year of DMO treatment and may serve as a valuable adjunctive to established procedures for retinal imaging in terms of individualised treatment plans.

Trial registration number NCT02554747

\section{INTRODUCTION}

Diabetic macular oedema (DMO) is the most common cause of visual impairment in people with diabetes and one of the leading causes of preventable vision loss in the working-age population of developed countries. ${ }^{1-3}$ The pathophysiology of DMO is not fully understood but involves both disruption of the blood-retinal barrier and inflammation

\section{Key messages}

What is already known about this subject?

Previous studies have demonstrated that the oxygen saturation increases with increasing severity of diabetic retinopathy (DR) with the highest saturations found in vision-threatening DR. Furthermore, pretreatment retinal oxygen saturation was recently demonstrated an independent predictor of visual acuity after treatment with inhibitors of vascular endothelial growth factor.

\section{What are the new findings?}

Our study demonstrated that higher retinal arteriolar oxygen saturation independently predicted the need for more intravitreal therapy during the first year of diabetic macular oedema treatment.

\section{How might these results change the focus of research or clinical practice? \\ - The results of this prospective clinical trial suggest that measurement of the oxygen saturation in retinal arterioles by retinal oximetry may serve as a valu- able adjunctive to already established procedures for retinal imaging in terms of individualised treat- ment plans.}

leading to capillary dropout, impaired vascular autoregulation and ultimately vascular leakage and macular oedema. ${ }^{24}$

Vascular endothelial growth factor (VEGF) is a key molecular mediator in DMO pathogenesis and a primary target of current DMO treatment. $^{2} 5$ VEGF upregulation is largely driven by retinal ischaemia and thus impaired retinal oxygen metabolism. Retinal vascular oxygen saturations can be evaluated non-invasively by dual-wavelength fundus photography (retinal oximetry) and serves as a marker of retinal oxygen metabolism. ${ }^{6}$ Using this method, the retinal venular oxygen saturation was demonstrated to increase with increasing severity of diabetic retinopathy (DR) to proliferative diabetic retinopathy (PDR) and DMO. ${ }^{78}$ These findings have been explained by alterations in retinal blood flow 
and a reduced extraction of oxygen to retinal tissue as a result of a loss of the capillary bed.

Pretreatment retinal arteriolar oxygen saturation was recently demonstrated an independent predictor of visual acuity (VA) after a loading dose of 3 monthly injections of ranibizumab. ${ }^{9}$ However, retinal oximetry is yet to be addressed as a marker of treatment load required even though it is pivotal with regards to translating the results into clinical practice and individualised treatment plans.

In a recently conducted 12-month randomised clinical trial of patients with centre involving DMO, we demonstrated that with similar functional outcome, combination therapy with intravitreal aflibercept and properly timed focal/grid laser photocoagulation approximately halved the need for intravitreal therapy during the first year of treatment as compared with earlier studies with aflibercept monotherapy. ${ }^{10}$ Still, the need for intravitreal therapy ranged from three to nine injections during the first year of treatment.

Hence, based on data from our clinical trial, we aimed to examine retinal vascular oxygen saturation as a non-invasive marker of treatment load of intravitreal aflibercept during the first year of treatment in patients with DMO.

\section{MATERIALS AND METHODS}

This study was based on data collected during the abovementioned 12-month randomised clinical trial and includes 35 eyes of 25 patients with centre involving DMO referred to Odense University Hospital, Denmark, between 1 October 2015 and 31 December 2017. ${ }^{10}$

Eligibility criteria were centre involving DMO, age 18-99 years, best corrected visual acuity (BCVA) between 35 and 80 Early Treatment Diabetic Retinopathy Study (ETDRS) letters and central retinal thickness (CRT) of more than $300 \mu \mathrm{m}$. We excluded patients who were pregnant, had active PDR, a history of panretinal photocoagulation or had been subjected to intraocular surgery (including focal/grid laser photocoagulation and intravitreal VEGF inhibition) within 4 months prior to inclusion.

Patients provided a full medical history at baseline (BL). Clinical examination included measurements of brachial arterial blood pressure (Omron 705CP, Hoofdrop, The Netherlands) and haemoglobin A1c (HbA1c; Tosoh G8, Alere, Holstebro, Denmark). Mean arterial pressure $(\mathrm{MAP})$ was calculated as $\mathrm{BP}_{\mathrm{d}}+\left(\mathrm{BP}_{\mathrm{s}}-\mathrm{BP}_{\mathrm{d}}\right) / 3$ where $\mathrm{BP}_{\mathrm{d}}$ is the diastolic blood pressure and $\mathrm{BP}_{\mathrm{s}}$ is the systolic blood pressure. Body mass index (BMI) was calculated as weight $(\mathrm{kg}) /$ height $(\mathrm{m}),{ }^{2}$ and smoking was quantified as pack years (one pack year was defined as 20 cigarettes/day for 1 year).

BCVA was assessed using ETDRS charts (Precision Vison, Illinois, USA) at a starting distance of $4 \mathrm{~m}$. Furthermore, ophthalmic examination included slit lamp and fundus biomicroscopy in mydriasis with tropicamide $10 \mathrm{mg} / \mathrm{mL}$ and phenylephrine $10 \%$, optical coherence tomography (OCT; 3D OCT-2000 Spectral domain OCT, Topcon, Tokyo, Japan) and $50^{\circ}$ macula-centred fundus fluorescein angiography (TRC-50DX fundus camera, Topcon).

Once consented, patients then received 3-monthly intravitreal injections of $2.0 \mathrm{mg}$ aflibercept followed by focal/grid laser photocoagulation using either Navilas (OD-OS GmbH, Teltow, Germany) or PASCAL laser (Optimedica Corp., Santa Clara, California, USA). From month 4 (M4) through month 12 (M12), patients were followed monthly and additional intravitreal injections were given pro re nata (PRN) if CRT increased by $>20 \%$ from the lowest measurement or loss in BCVA $>5$ ETDRS letters occurred as compared with BL.

As no differences between treatment arms of Navilas and Pascal laser photocoagulation were found in regards to functional outcome or need for intravitreal therapy, data from all patients were pooled for further analyses of retinal vascular oxygen saturations in this current study. ${ }^{10}$

\section{Retinal oximetry}

Retinal oxygen saturations were assessed using the Oxymap model T1 (Oxymap, Reykjavik, Iceland). The technique and equipment have been described in detail previously. ${ }^{6} 1112$ In brief, oxygen saturations are determined based on the intensity of reflected light from retinal vessels and surrounding tissue and rely on the light absorbance of oxyhaemoglobin and deoxyhaemoglobin. A dual-wavelength technique is used to calculate the relative concentrations of haemoglobin and oxyhaemoglobin and thereby oxygen saturations.

All measurements were performed in $50^{\circ}$ optic disc-centred images and graded in accordance with a prespecified protocol by a single-trained grader (SLB). Two concentric circles were semiautomatically placed around the optic disc. To avoid reflection of light from the optic disc, the inner circle was manually placed with a 20-30 pixels margin to the edge of the disc and the outer circle was automatically placed at three times the diameter of the inner circle. The four largest arterioles and venules representing each of the four quadrants were then automatically traced at a length between 50 and 200 pixels and oxygen saturations are presented as the average saturation of the traced arterioles and venules, respectively (figure 1). Retinal oximetry was performed at BL and measurements were repeated on the same vessel segments at month 12 . The grading process was masked in regards to the need for intravitreal therapy.

\section{Statistical analysis}

All statistical calculations were performed using STATA V.15.1 (StataCorp LLC) and figures were formatted using SigmaPlot V.14.0 (Systat Software, San Jose, California, USA). Continuous data are presented as mean and categorical data as per cent. Statistical differences were tested at a 0.05 level and $95 \%$ CI were estimated around point estimates where relevant. Differences between groups of eyes below (group A) and above (group B) the 50 


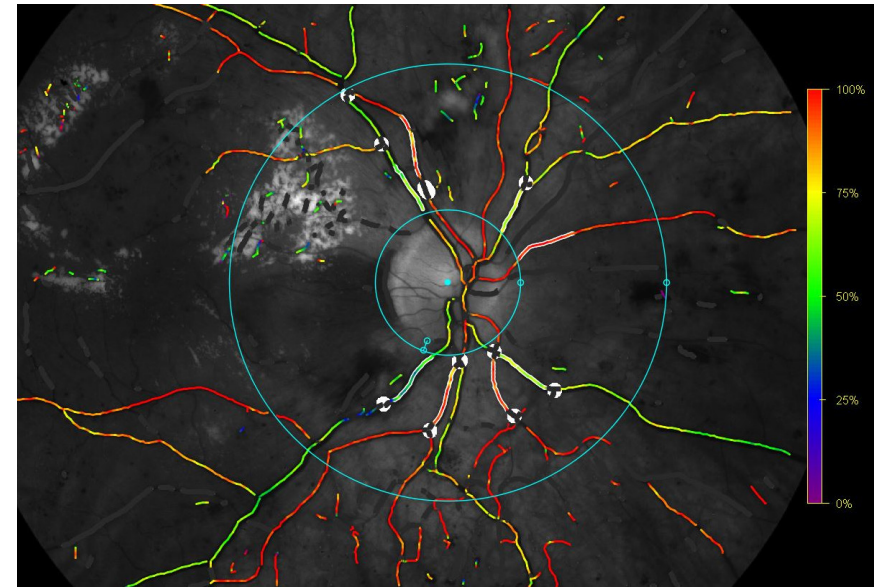

Figure 1 Retinal oximetry by Oxymap T1. Overlaid colour map indicates oxygen saturations in retinal vessels. The traced vessels representing the four retinal quadrants are highlighted within the concentric blue circles and excluded vessel segments are covered in white.

percentile of retinal arteriolar and venular oxygen saturations, respectively, were tested employing cluster robust standard errors for linear regression models as patients were allowed to participate with both eyes. A multiple linear regression model, also employing clustered-robust standard errors, was performed with retinal arteriolar and venular oxygen saturations as predictors of the number of injections during the first year of treatment (with adjustments for age, duration of diabetes, HbA1c, MAP, BCVA and CRT). The contribution of retinal oxygen saturation to explain the variation in need for treatment was finally examined in a univariate regression model applying $\mathrm{R}^{2}$.

Sample size was determined by a calculation of power comparing the mean of two independent samples (number of injections in groups A and B) that stated a required inclusion of minimum seven eyes in each group $\left(\alpha=0.05\right.$, power $=0.90$, mean $_{1}=4$ and mean $\left._{2}=6\right)$.

\section{Patient and public involvement}

Written informed consent was obtained from the subjects after explanation of the nature and possible consequences of the study. Patients or the public were not involved in the design, or conduct, or reporting, or dissemination of our research.

\section{RESULTS}

Forty-four eyes of 33 patients fulfilled the criteria of inclusion. One patient died during follow-up of reasons unrelated to the study. One was discontinued from the study as the patient was unable to follow scheduled visits due to other severe complications to diabetes. Another two were discontinued according to protocol at month 5 as eyes were not suitable for laser and hence, follow-up data were not available for this study. At last, additionally five eyes of four patients were excluded because of poor image quality due to cataract. This led to a final inclusion of 35 eyes of 25 patients.
Four were women (6 eyes) and 21 were men (29 eyes). Twenty-four had type 2 diabetes and one had type 1 diabetes. Five eyes of five patients had previously been subjected to focal/grid laser photocoagulation of which two had also previously received intravitreal VEGF inhibition. Mean HbA1c was 63.4 (55.1-71.8) $\mathrm{mmol} / \mathrm{mol}$ and mean age and duration of diabetes were 61.3 (56.0-66.7) and 11.2 (6.3-16.1) years, respectively. Mean systolic and diastolic blood pressure was 139.5 (132.3-146.7) and 82.9 (77.6-88.3) $\mathrm{mm} \mathrm{Hg}$ and thus, mean MAP was 101.8 (96.3107.3) mm Hg. Mean BMI was 28.4 (25.9-31.0) kg/m², and the mean number of pack years was 13.2 (5.4-21.0). Mean BCVA was 72.0 (68.9-75.1) ETDRS letters and mean CRT was 389.6 (355.5-423.7) $\mu \mathrm{m}$. Mean arteriolar and venular oxygen saturations were 95.7 (93.0-98.4)\% and 62.7 (59.4-65.9)\%. There were no differences in any BL parameters between eyes below (group A) and above (group B) the 50 percentile of retinal arteriolar or venular oxygen saturations, respectively (table 1 ).

From BL to month 12, BCVA improved by 8.7 (7.2-10.2) ETDRS letters. Likewise, there was a CRT reduction of 100.7 (68.2-133.3) $\mu \mathrm{m}$ and the mean number of needed injections was $4.3(3.8-4.8)$. There were no difference in retinal arteriolar or venular oxygen saturation between BL and month 12 (95.7 (93.0-98.4)\% vs 95.0 (92.3-97.7) $\%, \mathrm{p}=0.31$ and 62.7 (59.4-65.9) \% vs 61.8 (57.8-65.9) \%, $\mathrm{p}=0.57)$.

Eyes with a retinal arteriolar oxygen saturation above the 50 percentile (group B) had a higher need for intravitreal therapy from BL through M12 as compared with eyes with a retinal arteriolar oxygen saturation below the 50 percentile $(5.0(4.2-5.8)$ vs $3.6(3.1-4.0), \mathrm{p}=0.002)$ without differences in BCVA improvement and CRT reduction (9.2 (7.2-11-2) ETDRS letters vs 8.1 (5.8-10.4) ETDRS letters, $\mathrm{p}=0.49$ and 118.1 (63.0-173.2) $\mu \mathrm{m}$ vs 79.7 (49.4-109.9) $\mathrm{m}, \mathrm{p}=0.20)$. The difference in need for therapy remained statistically significant in a multiple linear regression model with groups of retinal arteriolar oxygen saturation as predictors of the number of injections during the first year of treatment (with adjustments for age, duration of diabetes, HbAlc, MAP, BCVA and CRT) (table 2). No difference in BCVA improvement or CRT reduction was found.

The distribution of eyes in groups of retinal arteriolar oxygen saturation accounted for approximately $19 \%$ of the variation in the need for intravitreal therapy. In the multiple regression model, all included parameters together explained approximately $48 \%$ of the variation in the need for intravitreal therapy.

There was no difference in the need for intravitreal therapy between groups of retinal venular oxygen saturation.

\section{DISCUSSION}

This study demonstrates that increased retinal arteriolar oxygen saturation significantly contributes to being able to predict a higher treatment requirement for intravitreal 
Table 1 Baseline characteristics of eyes according to retinal oxygen saturation

\begin{tabular}{|c|c|c|c|}
\hline & \multicolumn{2}{|c|}{ Groups according to percentiles of arteriolar oxygen saturation } & \multirow[b]{2}{*}{$P$ value } \\
\hline & Group A & Group B & \\
\hline Arteriolar oxygen saturation (\%) & $91.1(89.8-92.3)$ & $100.1(96.6-103.5)$ & \\
\hline$n$ & 17 & 18 & \\
\hline Age (years) & $64.0(55.5-72.5)$ & $58.8(53.5-64.0)$ & 0.25 \\
\hline Sex (\% women) & 17.7 & 16.7 & 0.94 \\
\hline Duration of diabetes (years) & $12.9(5.9-19.9)$ & $9.7(3.2-16.2)$ & 0.48 \\
\hline Haemoglobin A1c (mmol/mol) & $64.2(52.6-75.9)$ & $62.6(51.1-74.2)$ & 0.84 \\
\hline Body mass index $\left(\mathrm{kg} / \mathrm{m}^{2}\right)$ & $28.0(25.3-30.7)$ & $28.9(25.3-32.4)$ & 0.63 \\
\hline Mean arterial pressure $(\mathrm{mm} \mathrm{Hg})$ & $101.0(94.6-107.4)$ & $102.5(95.5-109.5)$ & 0.68 \\
\hline Smoking (pack years) & $12.2(2.2-22.1)$ & $14.2(4.2-24.3)$ & 0.73 \\
\hline Best corrected visual acuity (ETDRS letters) & $74.7(72.5-76.8)$ & $69.5(64.0-75.0)$ & 0.08 \\
\hline \multirow[t]{2}{*}{ Central retinal thickness $(\mu \mathrm{m})$} & $367.1(334.4-399.8)$ & 410.9 (349.6-472.2) & 0.20 \\
\hline & \multicolumn{2}{|c|}{$\begin{array}{l}\text { Groups according to percentiles of venular oxygen } \\
\text { saturation }\end{array}$} & \\
\hline Venular oxygen saturation (\%) & $56.4(53.8-59.0)$ & $69.3(66.6-71.9)$ & \\
\hline$n$ & 18 & 17 & \\
\hline Age (years) & $60.4(55.0-65.8)$ & $62.3(52.8-71.8)$ & 0.72 \\
\hline Sex (\% women) & 16.7 & 17.7 & 0.94 \\
\hline Duration of diabetes (years) & $14.4(7.6-21.1)$ & $7.9(1.2-14.6)$ & 0.18 \\
\hline Haemoglobin A1c (mmol/mol) & $66.0(56.4-75.6)$ & $60.6(46.3-74.8)$ & 0.52 \\
\hline Body mass index $\left(\mathrm{kg} / \mathrm{m}^{2}\right)$ & $28.1(25.3-30.8)$ & $28.8(25.0-32.6)$ & 0.72 \\
\hline Mean arterial pressure $(\mathrm{mm} \mathrm{Hg})$ & $101.6(94.6-108.5)$ & $102.0(95.2-108.8)$ & 0.91 \\
\hline Smoking (pack years) & $17.6(5.3-29.9)$ & $8.6(0.33-16.8)$ & 0.22 \\
\hline Best corrected visual acuity (ETDRS letters) & $73.1(69.8-76.3)$ & $70.9(65.7-76.1)$ & 0.46 \\
\hline Central retinal thickness $(\mu \mathrm{m})$ & $397.8(339.7-456.0)$ & 380.9 (346.8-414.9) & 0.61 \\
\hline
\end{tabular}

Continuous data presented as mean with $(95 \% \mathrm{Cls})$ and categorical data as per cent. Differences between groups of eyes with the lowest and highest retinal arteriolar oxygen saturations, respectively, were tested employing cluster robust standard errors for linear regression models.

ETDRS, Early Treatment Diabetic Retinopathy Study.

aflibercept during the first year of DMO treatment in a PRN regimen.

In accordance with previous studies, retinal venular oxygen saturation found in this study was higher in patients with DMO as compared with previously measured reference values for healthy individuals and remained unchanged during anti-VEGF treatment. ${ }^{911}$ The lack of reduction in the pathologically increased venular oxygen saturation towards normal levels may seem counterintuitive given the beneficial effects of intravitreal aflibercept on both functional and anatomical outcomes. However, the applied method of retinal oximetry may be insensitive to regional changes in retinal blood flow and oxygen saturations in the macular area as the oxygen saturation was based on measurements on the four largest arterioles and venules passing through a protocol-defined area surrounding the optic disc. Furthermore, the oximeter does not provide any data on absolute oxygen delivery to the retinal tissue, but rather a snapshot of vascular oxygen saturations at a given blood flow. Additionally, the relative contribution to oxygen delivery of retinal and choroidal vasculature may vary and especially around the macular area.

Previous data have shown that while early haemodynamic changes in the diabetic retina involves arteriolar constriction and hypoperfusion, a change towards enhanced blood flow and arteriolar hyperperfusion is seen in later stages of DR. This is presumably due to loss of autoregulatory mechanisms and release of dilatory factors (eg, VEGF) from the hypoxic retina. ${ }^{13-16}$ Hence, altered arteriolar blood flow may explain the measured variation in retinal arteriolar oxygen saturation. Furthermore, it may at the same time explain the differences in treatment load as increased arteriolar oxygen saturation due to hyperperfusion may also reflect a worse degree of DMO. It must be emphasised that the associations between blood flow, oxygen saturation and disease severity and stage of progression are not fully understood. 
Table 2 Regression coefficients, Cls and $\mathrm{p}$ values from multiple regression for the studied baseline parameters to predict the need for intravitreal aflibercept from baseline to month 12

\begin{tabular}{lccl}
\hline Variable & Coefficients & $\mathbf{9 5 \%} \mathbf{C l}$ & P value \\
\hline $\begin{array}{l}\text { Retinal arteriolar } \\
\text { oxygen saturation } \\
\text { (group A as } \\
\text { reference) }\end{array}$ & 1.38 & 0.53 to 2.23 & $0.003^{*}$ \\
\hline $\begin{array}{l}\text { Age } \\
\text { Duration of } \\
\text { diabetes }\end{array}$ & -0.01 & -0.04 to 0.03 & 0.64 \\
\hline $\begin{array}{l}\text { Haemoglobin A1c } \\
\text { Mean arterial }\end{array}$ & -0.01 & -0.01 to 0.07 & 0.11 \\
$\begin{array}{l}\text { pressure } \\
\text { Visual acuity }\end{array}$ & 0.02 & -0.04 to 0.02 & 0.43 \\
\hline $\begin{array}{l}\text { Central retinal } \\
\text { thickness }\end{array}$ & 0.01 & -0.00 to 0.02 & 0.18 \\
\hline \begin{tabular}{l} 
Intercept \\
\hline
\end{tabular} & 3.50 & -5.59 to 12.58 & 0.44 \\
\hline
\end{tabular}

${ }^{*}$ Indicates $\mathrm{p}<0.05$.

One may expect that variation in the oxygen saturation in retinal arterioles would be limited by a ceiling effect as fully saturated blood is led towards the retinal tissue. However, the blood in retinal arterioles is desaturated by approximately 5\%-10\% immediately after passage through the optic disc even in healthy individuals. The reduction is thought to be due to countercurrent exchange between the central retinal arteriole and venule as a consequence of close proximity within the optic nerve and leaves room for arteriolar oxygen saturation to increase alongside an increase in retinal blood flow. $^{6} 111718$

While the oxygen saturation in retinal venules has previously been demonstrated to increase with increasing severity of DR, we did not find any association between venular oxygen saturation and need for intravitreal therapy in our study on patients with DMO. ${ }^{7}$ The increase in venular oxygen saturation with increasing severity of DR has previously been explained by lower oxygen consumption due to retinal ischaemia and shunting of blood. ${ }^{79}$ Hence, the venular oxygen saturation may be closer associated with ischaemic changes in the peripheral retina, whereas DMO is more closely associated with changes in flow and perfusion in the macular area. ${ }^{19}$

Our demonstration of retinal arteriolar oxygen saturation as a predictor of treatment response is supported by recently published data that increased retinal arteriolar oxygen saturation also predicts good functional outcome in regards of VA. ${ }^{9}$

HbA1c, blood pressure and duration of diabetes are well recognised systemic risk factors of DMO development and progression of disease. ${ }^{220}$ Furthermore, VA and foveal thickness prior to treatment have been associated with functional and anatomical outcome, respectively. ${ }^{21}$ Hence, these parameters were included in our multiple linear regression model, even though systemic factors have so far been unable to predict treatment load of anti-VEGF treatment. ${ }^{22}$ Accordingly, the systemic factors were all together only able to explain approximately $12 \%$ of the variation in need for intravitreal therapy if the ocular parameters were experimentally removed from our regression model. Thus, our results concur with previous reports and emphasise the importance of intraocular parameters as predictors of treatment response in advanced stages of disease. It must, however, be emphasised that oxygen saturation in retinal arterioles only explained approximately $19 \%$ of the variation in need for intravitreal therapy.

Due to the limited number of patients in this study, we decided to exclude smoking (pack-years) from the regression model. A previous study on healthy individuals has demonstrated that neither arteriolar nor venular oxygen saturation differ between patients categorised as either current smokers or non-smokers. ${ }^{11}$ Furthermore, we did not find any difference in the number of pack years between groups $\mathrm{A}$ and $\mathrm{B}$ in either retinal arterioles or venules. In contrast, we speculate that the 'time from last cigarette' could potentially influence oximetry measurements. Thus, the oxygen saturation in facial skin has been demonstrated to decrease immediately after cigarette smoking. ${ }^{23}$

We allowed that patients included in this study could previously have been subjected to both focal/grid laser photocoagulation and anti-VEGF therapy if performed more than 4 months prior to inclusion. In contrast to the previously demonstrated effect of panretinal photocoagulation on the oxygen saturation in retinal vessels, we do not expect focal/grid laser photocoagulation to course any detectable effect on retinal oximetry measurements due to the very limited amount of affected retinal tissue. $^{2425}$

Likewise, given that current available anti-VEGF agents have a half-life of approximately 7-10 days in humans and an estimated range of VEGF suppression between 26 and 69 days, we do not believe that anti-VEGF therapy administered more than 4 months prior to inclusion will have had any impact on the oxygen saturations measured in this study. ${ }^{26}$ However, data concerning this subject are lacking and the fact that we did not find any change in retinal oxygen saturations between $\mathrm{BL}$ and month 12 after both anti-VEGF therapy and focal/grid laser photocoagulation may in truth represent the best available evidence to substantiate our assumption.

This study is unique from most previously conducted studies by addressing predictors of treatment load of intravitreal therapy rather than predictors of functional and anatomical outcome. We believe that this is essential in regards to translation of results into clinical practice and individualised treatment plans as retinal oximetry may assist clinicians to identify patients who are expected to have the highest need for therapy. Furthermore, this study was strengthened by the prospective design and well-defined treatment algorithm. 
A limitation to the study was the number of participants. However, sample size was calculated based on a predefined clinically meaningful difference given the relatively limited mean number of needed injections. Furthermore, the relatively short period of follow-up limits our study as our results do not provide data beyond 12 months of treatment. Given the nature of DMO and DMO treatment, long-term outcomes on retinal oximetry as a predictor of treatment load are warranted.

We also acknowledge that treatment algorithms for DMO vary greatly between clinical practices and hence, our results are so far limited to the specified PRN regimen. It is, thus, also unknown to what extend the adjunctive use of focal/grid laser photocoagulation has an impact on the ability to transfer our results to treatment regimens with aflibercept monotherapy. However, our results are approximately at the same level as previous anti-VEGF trials for DMO in regards to improvement in VA and CRT. ${ }^{27}$ Hence, we do not believe that the predictive value of retinal arteriolar oxygen saturation is unique to our population due to differences in response to treatment.

Also, the number of intravitreal injections between BL and month 12 in this study was fairly low as compared with the most previously conducted studies on antiVEGF monotherapy for DMO. ${ }^{27}$ Hence, we speculate that the difference in need for therapy between groups (A and B) may be more pronounced in other treatment regimens with more frequent intravitreal injections. However, further studies with other treatment strategies, for example, intravitreal aflibercept with and without adjunctive focal/grid laser photocoagulation or treat and extend regimens are warranted to clarify the generalisability of our results.

In conclusion, retinal arteriolar oxygen saturation contributes significantly to predict the need for intravitreal aflibercept during the first year of DMO treatment. Hence, retinal oximetry may serve as a valuable noninvasive adjunctive to the already established procedures for retinal imaging in terms of individualised treatment plans.

Acknowledgements The authors thank all funding organisations for the financial support for this study. Thanks to the Department of Ophthalmology, Odense University Hospital for providing the facilities and equipment necessary.

Contributors All authors contributed equally to the research design and manuscript preparation. Data acquisition/research execution and data analysis were carried out by SLB and JG. SLB and JG had full access to all the data in the study and all authors take responsibility for the integrity of the data and the accuracy of the data analysis.

Funding SLB reports grants from the University of Southern Denmark, grants from Odense University Hospital, grants from Fight for Sight Denmark, grants from The Synoptik Foundation and grants from The Danish Diabetes Academy supported by the Novo Nordisk Foundation, during the conduct of the study.

Competing interests SLB reports no competing interests. TP reports personal fees from Novartis, personal fees from Bayer, personal fees from OPTOS, personal fees from Heidelberg, outside the submitted work. JG reports personal fees from Bayer, personal fees from Novartis, outside the submitted work.

Patient consent for publication Not required.
Ethics approval This study was carried out in accordance with the tenets of the Declaration of Helsinki and Good clinical practice. Ethical approval was obtained from the Regional Scientific Ethical Committee for Southern Denmark (ID: S-20150077).

Provenance and peer review Not commissioned; externally peer reviewed.

Data availability statement Data are available upon request.

Open access This is an open access article distributed in accordance with the Creative Commons Attribution Non Commercial (CC BY-NC 4.0) license, which permits others to distribute, remix, adapt, build upon this work non-commercially, and license their derivative works on different terms, provided the original work is properly cited, appropriate credit is given, any changes made indicated, and the use is non-commercial. See: http://creativecommons.org/licenses/by-nc/4.0/.

ORCID iD

Søren Leer Blindbæk http://orcid.org/0000-0003-0628-684X

\section{REFERENCES}

1 Cheung N, Mitchell P, Wong TY. Diabetic retinopathy. Lancet 2010;376:124-36.

2 Tan GS, Cheung N, Simó R, et al. Diabetic macular oedema. Lancet Diabetes Endocrinol 2017;5:143-55.

3 Wong TY, Mwamburi M, Klein R, et al. Rates of progression in diabetic retinopathy during different time periods: a systematic review and meta-analysis. Diabetes Care 2009;32:2307-13.

4 Stitt AW, Curtis TM, Chen M, et al. The progress in understanding and treatment of diabetic retinopathy. Prog Retin Eye Res 2016;51:156-86.

5 Aiello LP, Avery RL, Arrigg PG, et al. Vascular endothelial growth factor in ocular fluid of patients with diabetic retinopathy and other retinal disorders. N Engl J Med 1994;331:1480-7.

6 Stefánsson E, Olafsdottir OB, Eliasdottir TS, et al. Retinal oximetry: metabolic imaging for diseases of the retina and brain. Prog Retin Eye Res 2019;70:1-22.

7 Jørgensen CM, Hardarson SH, Bek T. The oxygen saturation in retinal vessels from diabetic patients depends on the severity and type of vision-threatening retinopathy. Acta Ophthalmol 2014;92:34-9.

8 Hammer M, Vilser W, Riemer T, et al. Diabetic patients with retinopathy show increased retinal venous oxygen saturation. Graefes Arch Clin Exp Ophthalmol 2009;247:1025-30.

9 Bek T, Jørgensen CM. The systemic blood pressure and oxygen saturation in retinal arterioles predict the effect of intravitreal antiVEGF treatment on diabetic maculopathy. Invest Ophthalmol Vis Sci 2016;57:5429-34.

10 Blindbæk SL, Peto T, Grauslund J. Aflibercept and navigated versus conventional laser in diabetic macular oedema: a 12-month randomized clinical trial. Acta Ophthalmol 2019;97.

11 Geirsdottir A, Palsson O, Hardarson SH, et al. Retinal vessel oxygen saturation in healthy individuals. Invest Ophthalmol Vis Sci 2012;53:5433-42.

12 Palsson O, Geirsdottir A, Hardarson SH, et al. Retinal oximetry images must be standardized: a methodological analysis. Invest Ophthalmol Vis Sci 2012;53:1729-33.

13 Lockhart CJ, McCann AJ, Pinnock RA, et al. Multimodal functional and anatomic imaging identifies preclinical microvascular abnormalities in type 1 diabetes mellitus. Am J Physiol Heart Circ Physiol 2014;307:H1729-36.

14 Clermont AC, Aiello LP, Mori F, et al. Vascular endothelial growth factor and severity of nonproliferative diabetic retinopathy mediate retinal hemodynamics in vivo: a potential role for vascular endothelial growth factor in the progression of nonproliferative diabetic retinopathy. Am J Ophthalmol 1997;124:433-46.

15 Ciulla TA, Harris A, Latkany P, et al. Ocular perfusion abnormalities in diabetes. Acta Ophthalmol Scand 2002;80:468-77.

16 Grunwald JE, Riva CE, Brucker AJ, et al. Altered retinal vascular response to $100 \%$ oxygen breathing in diabetes mellitus. Ophthalmology 1984;91:1447-52.

17 Schweitzer D, Hammer M, Kraft J, et al. In vivo measurement of the oxygen saturation of retinal vessels in healthy volunteers. IEEE Trans Biomed Eng 1999;46:1454-65.

18 Bek T. Regional morphology and pathophysiology of retinal vascular disease. Prog Retin Eye Res 2013;36:247-59.

19 Bek T. Diabetic maculopathy caused by disturbances in retinal vasomotion. A new hypothesis. Acta Ophthalmol Scand 1999;77:376-80.

20 Early Treatment Diabetic Retinopathy Study Research Group. Fundus photographic risk factors for progression of diabetic 
retinopathy. ETDRS report number 12 . early treatment diabetic retinopathy study Research Group. Ophthalmology 1991;98:823-33.

21 Sophie R, Lu N, Campochiaro PA. Predictors of functional and anatomic outcomes in patients with diabetic macular edema treated with ranibizumab. Ophthalmology 2015;122:1395-401.

22 Wykoff CC, Elman MJ, Regillo CD, et al. Predictors of diabetic macular edema treatment frequency with ranibizumab during the open-label extension of the ride and rise trials. Ophthalmology 2016;123:1716-21.

23 Fan G-B, Wu P-L, Wang X-M. Changes of oxygen content in facial skin before and after cigarette smoking. Skin Res Technol 2012;18:511-5.
24 Jørgensen C, Bek T. Increasing oxygen saturation in larger retinal vessels after photocoagulation for diabetic retinopathy. Invest Ophthalmol Vis Sci 2014;55:5365-9.

25 Torp TL, Kawasaki R, Wong TY, et al. Changes in retinal venular oxygen saturation predict activity of proliferative diabetic retinopathy 3 months after panretinal photocoagulation. $\mathrm{Br} \mathrm{J}$ Ophthalmol 2018;102:383-7.

26 García-Quintanilla L, Luaces-Rodríguez A, Gil-Martínez M, et al. Pharmacokinetics of intravitreal anti-VEGF drugs in age-related macular degeneration. Pharmaceutics 2019;11:365.

27 Blindbaek SL, Peto T, Grauslund J. How do we evaluate the role of focal/grid photocoagulation in the treatment of diabetic macular edema? Acta Ophthalmol 2019;97:339-46. 